\title{
Finite simple groups and localization
}

\author{
José L. RODRÍGUEZ * \\ Departamento de Geometría, Topología y Química Orgánica \\ Universidad de Almería, E-04120 Almería, Spain \\ e-mail: jlrodri@ual.es \\ JÉRÔME SChereR ${ }^{\dagger}$ AND JACques ThÉvenaZ \\ Institut de Mathématiques, Université de Lausanne \\ CH-1015 Lausanne, Switzerland \\ e-mail: jerome.scherer@ima.unil.ch \\ e-mail: jacques.thevenaz@ima.unil.ch
}

\begin{abstract}
The purpose of this paper is to explore the concept of localization, which comes from homotopy theory, in the context of finite simple groups. We give an easy criterion for a finite simple group to be a localization of some simple subgroup and we apply it in various cases. Iterating this process allows us to connect many simple groups by a sequence of localizations. We prove that all sporadic simple groups (except possibly the Monster) and several groups of Lie type are connected to alternating groups. The question remains open whether or not there are several connected components within the family of finite simple groups.
\end{abstract}

\section{Introduction}

The concept of localization plays an important role in homotopy theory. The introduction by Bousfield of homotopical localization functors in Bou and more recently its popularization by Farjoun in [Far] has led to the study of localization functors in other categories. Special attention has been set on the category of groups $G r$, as the effect of a homotopical localization on the fundamental group is often best described by a localization functor L: $G r \rightarrow G r$.

*Partially supported by DGESIC grant PB97-0202 and the Swiss National Science Foundation.

${ }^{\dagger}$ Partially supported by the Swiss National Science Foundation. 
A localization functor is a pair (L, $\eta$ ) consisting of a functor L: $G r \rightarrow G r$ together with a natural transformation $\eta: \mathrm{Id} \rightarrow \mathrm{L}$ from the identity functor, such that $L$ is idempotent, meaning that the two morphisms $\eta_{\mathrm{L} G}, \mathrm{~L}\left(\eta_{G}\right): \mathrm{L} G \rightarrow \mathrm{LL} G$ coincide and are isomorphisms. A group homomorphism $\varphi: H \rightarrow G$ is called in turn a localization if there exists a localization functor $(\mathrm{L}, \eta)$ such that $G=\mathrm{L} H$ and $\varphi=\eta_{H}: H \rightarrow \mathrm{L} H$ (but we note that the functor $\mathrm{L}$ is not uniquely determined by $\varphi$ ). In this situation, we often say that $G$ is a localization of $H$. A very simple characterization of localizations can be given without mentioning localization functors: A group homomorphism $\varphi: H \rightarrow G$ is a localization if and only if $\varphi$ induces a bijection

$$
\varphi^{*}: \operatorname{Hom}(G, G) \cong \operatorname{Hom}(H, G)
$$

as mentionned in $\mathrm{Cas}$, Lemma 2.1]. In the last decade several authors (Casacuberta, Farjoun, Libman, Rodríguez) have directed their efforts towards deciding which algebraic properties are preserved under localization. An exhaustive survey about this problem is nicely exposed in Cas by Casacuberta. For example, any localization of an abelian group is again abelian. Similarly, nilpotent groups of class at most 2 are preserved, but the question remains open for arbitrary nilpotent groups. Finiteness is not preserved, as shown by the example $A_{n} \rightarrow S O(n-1)$ for $n \geq 10$ (this is the main result in [Lib1]). In fact, it has been shown by Göbel and Shelah in GS that any non-abelian finite simple group has arbitrarily large localizations (a previous version of this result, assuming the generalized continuum hypothesis, was obtained in [GRS]). In particular it is not easy to determine all possible localizations of a given object. Thus we restrict ourselves to the study of finite groups and wonder if it would be possible to understand the finite localizations of a given finite simple group. This paper is a first step in this direction.

Libman Lib2 observed recently that the inclusion $A_{n} \hookrightarrow A_{n+1}$ of alternating groups is a localization if $n \geq 7$. His motivation was to find a localization where new torsion elements appear (e.g. $A_{10} \hookrightarrow A_{11}$ is such a localization since $A_{11}$ contains elements of order 11). In these examples, the groups are simple, which simplifies considerably the verification of formula (0.1). It suffices to check if $\operatorname{Aut}(G) \cong \operatorname{Hom}(H, G)-\{0\}$.

This paper is devoted to the study of the behaviour of injective localizations with respect to simplicity. We first give a criterion for an inclusion of a simple group in a finite simple group to be a localization. We then find several infinite families of such localizations, for example $L_{2}(p) \hookrightarrow A_{p+1}$ for any prime $p \geq 13$ (cf. Proposition 2.3). Here $L_{2}(p)=P S L_{2}(p)$ is the projective special linear group. It is striking to notice that the three conditions that appear in our criterion for an inclusion of simple groups $H \hookrightarrow G$ to be a localization already appeared in the literature. For example the main theorem of KW2 states exactly that $J_{3} \hookrightarrow E_{6}(4)$ is a localization (see Section 3). Similarly the main theorem in [SWW] states that $S z(32) \hookrightarrow E_{8}(5)$ is a localization. Hence the language of 
localization theory can be useful to shortly reformulate some rather technical properties.

By Libman's result, the alternating groups $A_{n}$, for $n \geq 7$, are all connected by a sequence of localizations. We show that $A_{5} \hookrightarrow A_{6}$ is also a localization. A more curious way allows us to connect $A_{6}$ to $A_{7}$ by a zigzag of localizations:

$$
A_{6} \hookrightarrow T \hookrightarrow R u \hookleftarrow L_{2}(13) \hookrightarrow A_{14} \hookleftarrow \cdots \hookleftarrow A_{7}
$$

where $T$ is the Tits group, and $R u$ the Rudvalis group. This yields the concept of rigid component of a simple group. The idea is that among all inclusions $H \hookrightarrow G$, those that are localizations deserve our attention because of the "rigidity condition" imposed by (0.1): Any automorphism of $G$ is completely determined by its restriction to $H$. So, we say that two groups $H$ and $G$ lie in the same rigid component if $H$ and $G$ can be connected by a zigzag of inclusions which are all localizations.

Many finite simple groups can be connected to the alternating groups. Here is our main result:

Theorem The following finite simple groups all lie in the same rigid component:

(i) All alternating groups $A_{n}(n \geq 5)$.

(ii) The Chevalley groups $L_{2}(q)$ where $q$ is a prime power $\geq 5$.

(iii) The Chevalley groups $U_{3}(q)$ where $q$ is any prime power.

(iv) The Chevalley groups $G_{2}(p)$ where $p$ is an odd prime such that $(p+1,3)=1$.

(v) All sporadic simple groups, except possibly the Monster.

(vi) The Chevalley groups $L_{3}(3), L_{3}(5), L_{3}(11), L_{4}(3), U_{4}(2), U_{4}(3), U_{5}(2)$, $U_{6}(2), S_{4}(4), S_{6}(2), S_{8}(2), D_{4}(2),{ }^{2} D_{4}(2),{ }^{2} D_{5}(2),{ }^{3} D_{4}(2), D_{4}(3), G_{2}(2)^{\prime}$, $G_{2}(4), G_{2}(5), G_{2}(11), E_{6}(4), F_{4}(2)$, and $T={ }^{2} F_{4}(2)^{\prime}$.

The proof is an application of the localization criteria which are given in Sections 1 and 2 , but requires a careful checking in the ATLAS [CCN], or in the more complete papers about maximal subgroups of finite simple groups (e.g. [Kle, [LW], Wil2]). We do not know if the Monster can be connected to the alternating groups.

It is still an open problem to know how many rigid components of finite simple groups there are, even though our main theorem seems to suggest that there is only one. We note that the similar question for non-injective localizations has a trivial answer (see Section 1).

Let us finally mention that simplicity is not necessarily preserved by localization. This is the subject of the separate paper [RSV], where we exhibit for example a localization map from the Mathieu group $M_{11}$ to the double cover of the Mathieu group $M_{12}$. In our context this implies that the rigid component 
of a simple group may contain a non-simple group. This answers negatively a question posed both by Libman in Lib2 and Casacuberta in Cas] about the preservation of simplicity. In these papers it was also asked whether perfectness is preserved, but we leave this question unsolved.

Acknowledgments: We would like to thank Antonio Viruel, Jean Michel, as well as the referee for helpful comments. The first-named author also thanks the Institut de Mathématiques de l'Université de Lausanne for its kind hospitality and fantastic views of the Alps.

\section{A localization criterion}

Let us fix from now on a finite simple group $G$. In Theorem 1.4 below we list necessary and sufficient conditions for an inclusion $H \hookrightarrow G$ between two nonabelian finite simple groups to be a localization. These conditions are easier to deal with if the groups $H$ and $G$ satisfy some extra assumptions, as we show in the corollaries after the theorem. The proof is a variation of that of Corollary 4 in GRS.

We note here that we only deal with injective group homomorphisms because non-injective localizations abound. For example, for any two finite groups $G_{1}$ and $G_{2}$ of coprime orders, $G_{1} \times G_{2} \rightarrow G_{1}$ and $G_{1} \times G_{2} \rightarrow G_{2}$ are localizations. So the analogous concept of rigid component defined using non-injective localizations has no interest, since obviously any two finite groups are in the same component.

If the inclusion $i: H \hookrightarrow G$ is a localization, then so is the inclusion $H^{\prime} \hookrightarrow G$ for any subgroup $H^{\prime}$ of $G$ which is isomorphic to $H$. This shows that the choice of the subgroup $H$ among isomorphic subgroups does not matter.

Let $c: G \rightarrow \operatorname{Aut}(G)$ be the natural injection of $G$ defined as $c(g)=c_{g}: G \rightarrow G$, where $c_{g}$ is the inner automorphism given by $x \mapsto g x g^{-1}$. We shall always identify in this way a simple group $G$ with a subgroup of $\operatorname{Aut}(G)$ and the quotient $\operatorname{Out}(G)=\operatorname{Aut}(G) / G$ is then called the group of outer automorphisms of $G$.

Lemma 1.1 Let $G$ be a non-abelian simple group. Then the following diagram commutes

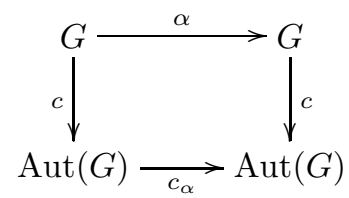

for any automorphism $\alpha \in \operatorname{Aut}(G)$.

Proof This is a trivial check. 
Lemma 1.2 Let $H$ be a non-abelian simple subgroup of a finite simple group $G$. Suppose that the inclusion $i: H \hookrightarrow G$ extends to an inclusion of their automorphism groups $i: \operatorname{Aut}(H) \hookrightarrow \operatorname{Aut}(G)$, i.e., the following diagram commutes

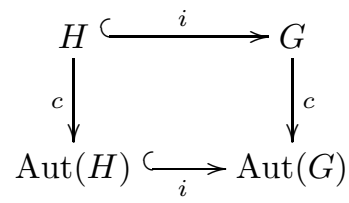

Then every automorphism $\alpha: H \rightarrow H$ extends to an automorphism $i(\alpha): G \rightarrow G$.

Proof We have to show that the following square commutes:

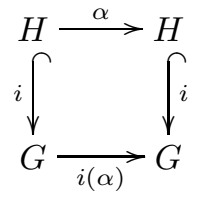

To do so we consider this square as the left-hand face of the cubical diagram

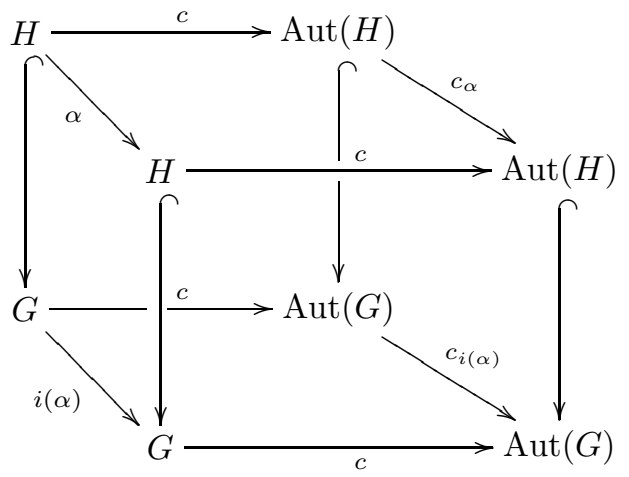

The top and bottom squares commute by Lemma 1.1. The front and back squares are the same and commute by assumption. The right-handsquare commutes as well because $i$ is a homomorphism. This forces the left-hand square to commute and we are done.

Remark 1.3 As shown by the preceding lemma, it is stronger to require that the inclusion $i: H \hookrightarrow G$ extends to an inclusion $i$ : $\operatorname{Aut}(H) \hookrightarrow \operatorname{Aut}(G)$ than to require that every automorphism of $H$ extends to an automorphism of $G$. In general we have an exact sequence

$$
1 \rightarrow C_{\mathrm{Aut}(G)}(H) \longrightarrow N_{\mathrm{Aut}(G)}(H) \longrightarrow \operatorname{Aut}(H)
$$


so the second condition is equivalent to the fact that this is a short exact sequence. However, in the presence of the condition $C_{\mathrm{Aut}(G)}(H)=1$, which plays a central role in this paper, we find that $N_{\text {Aut }(G)}(H) \cong$ Aut $(H)$. Thus any automorphism of $H$ extends to a unique automorphism of $G$, and this defines a homomorphism $i: \operatorname{Aut}(H) \hookrightarrow \operatorname{Aut}(G)$ extending the inclusion $H \hookrightarrow G$. Therefore, if the condition $C_{\operatorname{Aut}(G)}(H)=1$ holds, we have a converse of the above lemma and both conditions are equivalent. We will use the first in the statements of the following results, even though it is the stronger one. It is indeed easier to check in the applications.

Theorem 1.4 Let $H$ be a non-abelian simple subgroup of a finite simple group $G$ and let $i: H \hookrightarrow G$ be the inclusion. Then $i$ is a localization if and only if the following three conditions are satisfied:

1. The inclusion $i: H \hookrightarrow G$ extends to an inclusion $i: \operatorname{Aut}(H) \hookrightarrow \operatorname{Aut}(G)$.

2. Any subgroup of $G$ which is isomorphic to $H$ is conjugate to $H$ in $\operatorname{Aut}(G)$.

3. The centralizer $C_{\operatorname{Aut}(G)}(H)=1$.

Proof If $i$ is a localization, all three conditions have to be satisfied. Indeed given an automorphism $\alpha \in \operatorname{Aut}(H)$, formula (0.1) tells us that there exists a unique group homomorphism $\beta: G \rightarrow G$ such that $\beta \circ i=i \circ \alpha$. Since $G$ is finite and simple, $\beta$ is an automorphism. We set then $i(\alpha)=\beta$ and condition (1) follows. Given a subgroup $j: H^{\prime} \hookrightarrow G$ and an isomorphism $\phi: H \rightarrow H^{\prime}$, a similar argument with formula (0.1) ensures the existence of an automorphism $\beta \in \operatorname{Aut}(G)$ such that $\beta \circ i=j \circ \phi$. Thus condition (2) holds. Finally condition (3) is also valid since the unique extension of $1_{H}$ to $G$ is the identity.

Assume now that all three conditions hold. For any given homomorphism $\varphi: H \rightarrow G$, we need a unique homomorphism $\Phi: G \rightarrow G$ such that $\Phi \circ i=\varphi$. If $\varphi$ is trivial, we choose of course the trivial homomorphism $\Phi: G \rightarrow G$. It is unique since $H$ is in the kernel of $\Phi$, which must be equal to $G$ by simplicity. Hence, we can suppose that $\varphi$ is not trivial. Since $H$ is simple we have that $\varphi(H) \leq G$ and $H \cong \varphi(H)$.

By (2) there is an automorphism $\alpha \in \operatorname{Aut}(G)$ such that $c_{\alpha}(\varphi(H))=H$, or equivalently by Lemma 1.1, $\alpha(\varphi(H))=H$. Therefore the composite map

$$
H \stackrel{\varphi}{\longrightarrow} \varphi(H) \stackrel{\left.\alpha\right|_{\varphi(H)}}{\longrightarrow} H
$$

is some automorphism $\beta$ of $H$. By condition (1) this automorphism of $H$ extends to an automorphism $i(\beta): G \rightarrow G$. That is, the following square commutes:

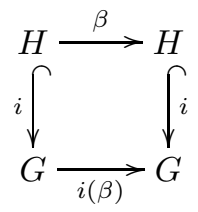


The homomorphism $\Phi=\alpha^{-1} i(\beta)$ extends $\varphi$ as desired. We prove now it is unique. Suppose that $\Phi^{\prime}: G \rightarrow G$ is a homomorphism such that $\Phi^{\prime} \circ i=\varphi$. Then, since $G$ is simple, $\Phi^{\prime} \in \operatorname{Aut}(G)$. The composite $\Phi^{-1} \Phi^{\prime}$ is an element in the centralizer $C_{\operatorname{Aut}(G)}(H)$, which is trivial by (3). This finishes the proof of the theorem.

Remark 1.5 The terminology used for condition (2) is that two subgroups $H$ and $H^{\prime}$ in $G$ fuse in $\operatorname{Aut}(G)$ if there is an automorphism $\alpha \in \operatorname{Aut}(G)$ such that $\alpha(H)=H^{\prime}$. Assuming condition (1) in Theorem 1.4 we have a short exact sequence

$$
1 \rightarrow C_{\operatorname{Aut}(G)}(H) \longrightarrow N_{\operatorname{Aut}(G)}(H) \longrightarrow \operatorname{Aut}(H) \rightarrow 1
$$

(compare with Remark 1.3). Moreover there are $\frac{|\operatorname{Aut}(G)|}{\left|N_{\operatorname{Aut}(G)}(H)\right|}$ subgroups in the conjugacy class of $H$ in $\operatorname{Aut}(G)$ and $\frac{|G|}{\left|N_{G}(H)\right|}$ subgroups in the conjugacy class of $H$ in $G$. If condition (2) also holds, this implies that the total number of conjugacy classes of subgroups isomorphic to $H$ in $G$ is equal to

$$
\frac{|\operatorname{Aut}(G)|}{\left|N_{\operatorname{Aut}(G)}(H)\right|} \cdot \frac{\left|N_{G}(H)\right|}{|G|}=\frac{|\operatorname{Out}(G)| \cdot|G|}{|\operatorname{Aut}(H)| \cdot\left|C_{\operatorname{Aut}(G)}(H)\right|} \cdot \frac{\left|N_{G}(H)\right|}{|G|}
$$

Condition (3) is thus equivalent to the following one, which is sometimes easier to verify:

3'. The number of conjugacy classes of subgroups of $G$ isomorphic to $H$ is equal to

$$
\frac{|\operatorname{Out}(G)|}{|\operatorname{Out}(H)|} \cdot \frac{\left|N_{G}(H)\right|}{|H|}
$$

We obtain immediately the following corollaries. Using the terminology in [Rot, p.158], recall that a group is complete if it has trivial centre and every automorphism is inner.

Corollary 1.6 Let $H$ be a non-abelian simple subgroup of a finite simple group $G$ and let $i: H \hookrightarrow G$ be the inclusion. Assume that $H$ and $G$ are complete groups. Then $i$ is a localization if and only if the following two conditions are satisfied:

1. Any subgroup of $G$ which is isomorphic to $H$ is conjugate to $H$.

2. $C_{G}(H)=1$.

The condition $C_{G}(H)=1$ is here equivalent to $N_{G}(H)=H$. This is often easier to check. It is in particular always the case when $H$ is a maximal subgroup of $G$. This leads us to the next corollary. 
Corollary 1.7 Let $H$ be a non-abelian simple subgroup of a finite simple group $G$ and let $i: H \hookrightarrow G$ be the inclusion. Assume that $H$ is a maximal subgroup of $G$. Then $i$ is a localization if and only if the following three conditions are satisfied:

1. The inclusion $i: H \hookrightarrow G$ extends to an inclusion $i$ : $\operatorname{Aut}(H) \hookrightarrow \operatorname{Aut}(G)$.

2. Any subgroup of $G$ which is isomorphic to $H$ is conjugate to $H$ in $\operatorname{Aut}(G)$.

3. There are $\frac{|\operatorname{Out}(G)|}{|\operatorname{Out}(H)|}$ conjugacy classes of subgroups isomorphic to $H$ in $G$.

Proof Since $H$ is a maximal subgroup of $G, N_{G}(H)=H$. The corollary is now a direct consequence of Theorem 1.4 taking into account Remark 1.5 about the number of conjugacy classes of subgroups of $G$ isomorphic to $H$.

\section{Localization in alternating groups}

We describe in this section a method for finding localizations of finite simple groups in alternating groups. Let $H$ be a simple group and $K$ a subgroup of index $n$. The (left) action of $H$ on the cosets of $K$ in $H$ defines a permutation representation $H \rightarrow S_{n}$ as in [Rot, Theorem 3.14, p.53]. The degree of the representation is the number $n$ of cosets. As $H$ is simple, this homomorphism is actually an inclusion $H \hookrightarrow A_{n}$. Recall that $\operatorname{Aut}\left(A_{n}\right)=S_{n}$ if $n \geq 7$.

Theorem 2.1 Let $H$ be a non-abelian finite simple group and $K$ a maximal subgroup of index $n \geq 7$. Suppose that the following two conditions hold:

1. The order of $K$ is maximal (among all maximal subgroups).

2. Any subgroup of $H$ of index $n$ is conjugate to $K$.

Then the permutation representation $H \hookrightarrow A_{n}$ is a localization.

Proof We show that the conditions of Theorem 1.4 are satisfied, starting with condition (1). Since $K$ is maximal, it is self-normalizing and therefore the action of $H$ on the cosets of $K$ is isomorphic to the conjugation action of $H$ on the set of conjugates of $K$. By our second assumption, this set is left invariant under $\operatorname{Aut}(H)$. Thus the action of $H$ extends to $\operatorname{Aut}(H)$ and this yields the desired extension $\operatorname{Aut}(H) \rightarrow S_{n}=\operatorname{Aut}\left(A_{n}\right)$.

To check condition (2) of Theorem 1.4, let $H^{\prime}$ be a subgroup of $A_{n}$ which is isomorphic to $H$ and denote by $\alpha: H \rightarrow H^{\prime}$ an isomorphism. Let $J$ be the stabilizer of a point in $\{1, \ldots, n\}$ under the action of $H^{\prime}$. Since the orbit of this point has cardinality $\leq n$, the index of $J$ is at most $n$, hence equal to $n$ by our first assumption. Thus $H^{\prime}$ acts transitively. So $H$ has a second transitive action via $\alpha$ and the action of $H^{\prime}$. For this action, the stabilizer of a point is a subgroup of $H$ of index $n$, hence conjugate to $K$ by assumption. So $K$ is also 
the stabilizer of a point for this second action and this shows that this action of $H$ is isomorphic to the permutation action of $H$ on the cosets of $K$, that is, to the first action. It follows that the permutation representation $H \stackrel{\alpha}{\longrightarrow} H^{\prime} \hookrightarrow A_{n}$ is conjugate in $S_{n}$ to $H \hookrightarrow A_{n}$.

Finally, since $H$ is a transitive subgroup of $S_{n}$ with maximal stabilizer, the centralizer $C_{S_{n}}(H)$ is trivial by $[\mathrm{DM}$, Theorem $4.2 \mathrm{~A}$ (vi)] and thus condition (3) of Theorem 1.4 is satisfied.

Among the twenty-six sporadic simple groups, twenty have a subgroup which satisfies the conditions of Theorem 2.1.

Corollary 2.2 The following inclusions are localizations:

$M_{11} \hookrightarrow A_{11}, M_{22} \hookrightarrow A_{22}, M_{23} \hookrightarrow A_{23}, M_{24} \hookrightarrow A_{24}, J_{1} \hookrightarrow A_{266}, J_{2} \hookrightarrow A_{100}$,

$J_{3} \hookrightarrow A_{6156}, J_{4} \hookrightarrow A_{173067389}, H S \hookrightarrow A_{100}, M c L \hookrightarrow A_{275}, C o_{1} \hookrightarrow A_{98280}$,

$\mathrm{Co}_{2} \hookrightarrow A_{2300}, \mathrm{Co}_{3} \hookrightarrow A_{276}, \mathrm{Suz} \hookrightarrow A_{1782}, \mathrm{He} \hookrightarrow A_{2058}, \mathrm{Ru} \hookrightarrow A_{4060}$,

$F i_{22} \hookrightarrow A_{3510}, F_{23} \hookrightarrow A_{31671}, H N \hookrightarrow A_{1140000}, L y \hookrightarrow A_{8835156}$.

Proof In each case, it suffices to check in the ATLAS [CCN] that the conditions of Theorem 2.1 are satisfied. It is however necessary to check the complete list of maximal subgroups in [KPW] for the Fischer group $F i_{23}$ and KW1] for the Janko group $J_{4}$.

We obtain now two infinite families of localizations. The classical projective special linear groups $L_{2}(q)=P S L_{2}(q)$ of type $A_{1}(q)$, as well as the projective special unitary groups $U_{3}(q)=P S U_{3}(q)$ of type ${ }^{2} A_{2}(q)$, are almost all connected to an alternating group by a localization. Recall that the notation $L_{2}(q)$ is used only for the simple projective special linear groups, that is if the prime power $q \geq 4$. Similarly the notation $U_{3}(q)$ is used for $q>2$.

Proposition 2.3 (i) The permutation representation $L_{2}(q) \hookrightarrow A_{q+1}$ induced by the action of $S L_{2}(q)$ on the projective line is a localization for any prime power $q \notin\{4,5,7,9,11\}$.

(ii) The permutation representation $U_{3}(q) \hookrightarrow A_{q^{3}+1}$ induced by the action of $S_{3}(q)$ on the set of isotropic points in the projective plane is a localization for any prime power $q \neq 5$.

Proof We prove both statements at the same time. The group $L_{2}(q)$ acts on the projective line, whereas $U_{3}(q)$ acts on the set of isotropic points in the projective plane. In both cases, let $B$ be the stabilizer of a point for this action (Borel subgroup). Let us also denote by $G$ either $L_{2}(q)$ or $U_{3}(q)$, where $q$ is a prime power as specified above, and $r$ is $q+1$, or $q^{3}+1$ respectively. Then $B$ is a subgroup of $G$ of index $r$ by Hup, Satz II-8.2] and Hup, Satz II-10.12].

By Hup, Satz II-8.28], which is an old theorem of Galois when $q$ is a prime, the group $L_{2}(q)$ has no non-trivial permutation representation of degree less than $r$ 
if $q \notin\{4,5,7,9,11\}$. The same holds for $U_{3}(q)$ by Coo, Table 1$]$ if $q \neq 5$. Thus $B$ satisfies condition (1) of Theorem 2.1.

It remains to show that condition (2) is also satisfied. The subgroup $B$ is the normalizer of a Sylow $p$-subgroup $U$, and $B=U T$, where $T$ is a complement of $U$ in $B$. If $N$ denotes the normalizer of $T$ in $G$, we know that $G=U N U$. This is the Bruhat decomposition (for more details see [Car, Chapter 8]). We are now ready to prove that any subgroup of $G$ of index $r$ is conjugate to $B$. Let $H$ be such a subgroup. It contains a Sylow $p$-subgroup, and we can thus assume it actually contains $U$. Since $G$ is generated by $U$ and $N$, the subgroup $H$ is generated by $U$ and $N \cap H$. Assume $H$ contains an element $x \in N-T$. The Weyl group $N / T$ is cyclic of order two, generated by the class of $x$ (the linear group $L_{2}(q)$ is a Chevalley group of type $A_{1}$ and the unitary group $U_{3}(q)$ is a twisted Chevalley group of type $\left.{ }^{2} A_{2}\right)$. Moreover $G=\left\langle U, x U x^{-1}\right\rangle$ by GLS, Theorem 2.3.8 (e)] and both $U$ and its conjugate $x U x^{-1}$ are contained in $H$. This is impossible because $H \neq G$, so $N \cap H=T \cap H$. It follows that $H$ is contained in $\langle U, T\rangle=B$. But $H$ and $B$ have the same order and therefore $H=B$.

Remark 2.4 This proof does not work for the action of $L_{n+1}(q)$ on the $n$ dimensional projective space if $n \geq 2$, because there is a second action of the same degree, namely the action on the set of all hyperplanes in $\left(\mathbb{F}_{q}\right)^{n+1}$. Thus there is another conjugacy class of subgroups of the same index, so condition (1) does not hold.

\section{Proof of the main theorem}

In order to prove our main theorem, we have to check that any group of the list is connected to an alternating group by a zigzag of localizations. When no specific proof is indicated for an inclusion to be a localization, it means that all the necessary information for checking conditions (1)-(3) of Theorem 1.4 is available in the ATLAS [CCN]. By $C_{2}$ we denote the cyclic group of order 2 .

\section{(i) Alternating groups.}

The inclusions $A_{n} \hookrightarrow A_{n+1}$, for $n \geq 7$, studied by Libman in Lib2, Example 3.4] are localizations by Corollary 1.7, with $\operatorname{Out}\left(A_{n}\right) \cong C_{2} \cong \operatorname{Out}\left(A_{n+1}\right)$. The inclusion $A_{5} \hookrightarrow A_{6}$ is a localization as well, since we have $\operatorname{Out}\left(A_{6}\right) \cong\left(C_{2}\right)^{2}$, $\operatorname{Out}\left(A_{5}\right) \cong C_{2}$, and there are indeed two conjugacy classes of subgroups of $A_{6}$ isomorphic to $A_{5}$ with fusion in $\operatorname{Aut}\left(A_{6}\right)$. The inclusion $A_{6} \hookrightarrow A_{7}$ is not a localization, but we can connect these two groups via a zigzag of localizations, for example as follows:

$$
A_{6} \hookrightarrow T \hookrightarrow R u \hookleftarrow L_{2}(13) \hookrightarrow A_{14}
$$

where $T$ denotes the Tits group, $R u$ the Rudvalis group and the last arrow is a localization by Proposition 2.3 . 
(ii) Chevalley groups $L_{2}(q)$.

By Proposition 2.3, all but five linear groups $L_{2}(q)$ are connected to an alternating group. The groups $L_{2}(4)$ and $L_{2}(5)$ are isomorphic to $A_{5}$, and $L_{2}(9) \cong A_{6}$. We connect $L_{2}(7)$ to $A_{28}$ via a chain of two localizations

$$
L_{2}(7) \hookrightarrow U_{3}(3)=G_{2}(2)^{\prime} \hookrightarrow A_{28}
$$

where we use Theorem 2.1 for the second map. Similarly, we connect $L_{2}(11)$ to $A_{22}$ via the Mathieu group $M_{22}$, using Corollary 2.2:

$$
L_{2}(11) \hookrightarrow M_{22} \hookrightarrow A_{22} .
$$

(iii) Chevalley groups $U_{3}(q)$.

For $q \neq 5$, we have seen in Proposition 2.3 (ii) that $U_{3}(q) \hookrightarrow A_{q^{3}+1}$ is a localization. One checks in [CCN, p.34] that there is a localization $A_{7} \hookrightarrow U_{3}(5)$, which connects $U_{3}(5)$ to the alternating groups.

(iv) Chevalley groups $G_{2}(p)$.

When $p$ is an odd prime such that $(p+1,3)=1$, we will see in Proposition 4.3 that $U_{3}(p) \hookrightarrow G_{2}(p)$ is a localization. We can conclude by (iii), since 5 is not a prime in the considered family.

(v) Sporadic simple groups.

By Corollary 2.2, we already know that twenty sporadic simple groups are connected with some alternating group. We now show how to connect all the other sporadic groups, except the Monster for which we do not know what happens.

For the Mathieu group $M_{12}$, we note that the inclusion $M_{11} \hookrightarrow M_{12}$ is a localization because there are two conjugacy classes of subgroups of $M_{12}$ isomorphic to $M_{11}$ (of index 11) with fusion in $\operatorname{Aut}\left(M_{12}\right)$ (cf. [CCN, p.33]). We conclude by Corollary 1.7.

The list of all maximal subgroups of $F i_{24}^{\prime}$ is given in $[\mathrm{LW}$ and one applies Theorem 1.4 to show that $H e \hookrightarrow F i_{24}^{\prime}$ is a localization (both groups have $C_{2}$ as group of outer automorphisms).

Looking at the complete list of maximal subgroups of the Baby Monster $B$ in Wil2, we see that $F i_{23} \hookrightarrow B$ is a localization, as well as $T h \hookrightarrow B, H N \hookrightarrow B$, and $L_{2}(11) \hookrightarrow B$ (see Proposition 4.1 in Wil2]). This connects Thompson's group $T h$ and the Baby Monster (as well as the Harada-Norton group HN) to the Fischer groups and also to the Chevalley groups $L_{2}(q)$.

Finally we consider the O'Nan group $O^{\prime} N$. By [Will, Proposition 3.9] we see that $M_{11} \hookrightarrow O^{\prime} N$ satisfies conditions (1)-(3) of Corollary 1.7 and thus is a localization. 
(vi) Other Chevalley groups.

The construction of the sporadic group Suz provides a sequence of five graphs (the Suzuki chain) whose groups of automorphisms are successively $\operatorname{Aut}\left(L_{2}(7)\right.$ ), $\operatorname{Aut}\left(G_{2}(2)^{\prime}\right)$, Aut $\left(J_{2}\right)$, Aut $\left(G_{2}(4)\right)$ and $\operatorname{Aut}(S u z)$ (see [Gor, p.108-9]). Each one of these five groups is an extension of $C_{2}$ by the appropriate finite simple group. All arrows in the sequence

$$
L_{2}(7) \hookrightarrow G_{2}(2)^{\prime} \hookrightarrow J_{2} \hookrightarrow G_{2}(4) \hookrightarrow S u z
$$

are thus localizations by Corollary 1.7 because they are actually inclusions of the largest maximal subgroup (cf. $[\overline{C C N}]$ ). This connects the groups $G_{2}(2)^{\prime}$ and $G_{2}(4)$ to alternating groups since we already know that $S u z$ is connected to $A_{1782}$ by Corollary 2.2. Alternatively, note that $G_{2}(4) \hookleftarrow L_{2}(13) \hookrightarrow A_{14}$ are localizations, using Proposition 2.3 for the second one.

The Suzuki group provides some more examples of localizations: $L_{3}(3) \hookrightarrow S u z$ by [WS, Section 6.6], and $U_{5}(2) \hookrightarrow S u z$ by [WS, Section 6.1]. We also have localizations

$$
A_{9} \hookrightarrow D_{4}(2)=O_{8}^{+}(2) \hookrightarrow F_{4}(2) \hookleftarrow{ }^{3} D_{4}(2)
$$

which connect these Chevalley groups (see Proposition 4.4 for the last arrow). We are able to connect three symplectic groups since $A_{8} \hookrightarrow S_{6}(2)$ and $S_{4}(4) \hookrightarrow H e$ are localizations, as well as $S_{8}(2) \hookrightarrow A_{120}$ by Theorem 2.1. This allows us in turn to connect more Chevalley groups as $U_{4}(2) \hookrightarrow S_{6}(2)$, and $O_{8}^{-}={ }^{2} D_{4}(2) \hookrightarrow S_{8}(2)$ are all localizations.

Each of the following localizations involves a linear group and connects some new group to the component of the alternating groups:

$L_{2}(11) \hookrightarrow U_{5}(2), L_{3}(3) \hookrightarrow T, L_{2}(7) \hookrightarrow L_{3}(11)$, and $L_{4}(3) \hookrightarrow F_{4}(2)$.

The localization $U_{3}(3) \hookrightarrow G_{2}(5)$ connects $G_{2}(5)$ and thus $L_{3}(5)$ by Proposition 4.2 below. Likewise, since we just showed above that $L_{3}(11)$ belongs to the same rigid component, then so does $G_{2}(11)$.

Next $M_{22} \hookrightarrow U_{6}(2)$ and $A_{12} \hookrightarrow O_{10}^{-}={ }^{2} D_{5}(2)$ are also localizations.

In the last three localizations, connecting the groups $U_{4}(3), E_{6}(4)$, and $D_{4}(3)$, the orders of the outer automorphism groups is larger than 2. Nevertheless, Theorem 1.4 applies easily. There is a localization $A_{7} \hookrightarrow U_{4}(3)$. There are four conjugacy classes of subgroups of $U_{4}(3)$ isomorphic to $A_{7}$, all of them being maximal. The dihedral group $D_{8} \cong \operatorname{Out}\left(U_{4}(3)\right)$ acts transitively on those classes and $S_{7}$ is contained in $\operatorname{Aut}\left(U_{4}(3)\right)$ (see [CCN, p.52]).

We have also a localization $J_{3} \hookrightarrow E_{6}(4)$. Here $\operatorname{Out}\left(E_{6}(4)\right) \cong D_{12}$ and there are exactly six conjugacy classes of sugroups isomorphic to $J_{3}$ in $E_{6}(4)$ which are permuted transitively by $D_{12}$. This is exactly the statement of the main theorem of $\mathrm{KW} 2$.

Finally $D_{4}(2) \hookrightarrow D_{4}(3)$ is a localization. Here we have $\operatorname{Out}\left(D_{4}(2)\right) \cong S_{3}$ and $\operatorname{Out}\left(D_{4}(3)\right) \cong S_{4}$. There are four conjugacy classes of subgroups of $D_{4}(3)$ isomorphic to $D_{4}(2)$. 


\section{Other localizations}

In this section, we give further examples of localizations between simple groups. We start with three infinite families of localizations. Except the second family, we do not know if the groups belong to the rigid component of alternating groups.

Lemma 4.1 Let $p$ be an odd prime with $(3, p-1)=1$. If $H \leq M \leq G_{2}(p)$ are subgroups with $H \cong L_{3}(p)$ and $M$ maximal, then $M \cong \operatorname{Aut}\left(L_{3}(p)\right)$.

Proof The order of $L_{3}(p)$ is $p^{3}\left(p^{3}-1\right)\left(p^{2}-1\right)$, which is larger than $p^{6}$. So the main theorem in LS implies that any maximal sugbroup of $G_{2}(p)$ containing $H$ has to be one of [LS, Table 1, p.300] or a parabolic subgroup. Of course $\left|L_{3}(p)\right|$ has to divide $|M|$. Therefore $M$ can not be parabolic because the order of a parabolic subgroup of $G_{2}(p)$ is $p^{6}\left(p^{2}-1\right)(p-1)$ (see [Kle, Theorem A]). When $p \neq 3$ there are only two maximal subgroups left to deal with. When $p=3$, we could also have used the ATLAS [CCN, p.60]. In both cases the only maximal subgroup whose order is divisible by $\left|L_{3}(p)\right|$ is isomorphic to $\operatorname{Aut}\left(L_{3}(p)\right)$.

Proposition 4.2 Let $p$ be an odd prime with $(3, p-1)=1$. Then there is a localization $L_{3}(p) \hookrightarrow G_{2}(p)$.

PROOF The strategy is to verify conditions (1)-(3) of Theorem 1.4, or rather conditions (1), (2), and (3') of Remark 1.5. Let us first review some facts from [Kle, Proposition 2.2]. There exist two subgroups $L_{+} \leq K_{+} \leq G_{2}(p)$ with $L_{+} \cong L_{3}(p)$ a subgroup of index 2 in $K_{+}$. This is the inclusion we consider here. Moreover $K_{+}=N_{G_{2}(p)}\left(L_{+}\right)$(this is Step 3 in [Kle, Proposition 2.2]), and $N_{\mathrm{Aut}\left(G_{2}(p)\right)}\left(L_{+}\right) \cong \operatorname{Aut}\left(L_{3}(p)\right)$ (Step 1). Thus $K_{+} \cong \operatorname{Aut}\left(L_{3}(p)\right)$ and condition (1) of Theorem 1.4 obviously holds. To check condition (2) consider a subgroup $H \leq G_{2}(p)$ with $H \cong L_{3}(p)$. By the above lemma $H$ must be contained in a maximal subgroup isomorphic to $\operatorname{Aut}\left(L_{3}(p)\right)$. When $p \neq 3$ the group $G_{2}(p)$ is complete and [Kle, Theorem A] shows that $H$ is contained in some conjugate of $K_{+}$. Therefore $H$ is conjugate to $L_{+}$in $G_{2}(p)$. When $p=3$, the group $G_{2}(p)$ is not complete. The group $\operatorname{Out}\left(G_{2}(3)\right)$ is cyclic of order 2 as there is a "graph automorphism" $\gamma: G_{2}(3) \rightarrow G_{2}(3)$ which is not an inner automorphism (we follow the notation from [Kle, Proposition $2.2(\mathrm{v})]$ ). Then $K_{+}$and $\gamma\left(K_{+}\right)$ are not conjugate in $G_{2}(3)$, but of course they are in Aut $\left(G_{2}(3)\right)$. Again Theorem A in [Kl]] shows that $H$ is conjugate either to $L_{+}$or to $\gamma\left(L_{+}\right)$in $G_{2}(3)$ and condition (2) is also satisfied. Finally condition (3') is valid since $\operatorname{Out}\left(L_{3}(p)\right)$ is cyclic of order 2. The number of conjugacy classes of subgroups isomorphic to $L_{3}(p)$ in $G_{2}(p)$ is 1 when $p \neq 3$ and 2 when $p=3$.

Proposition 4.3 Let $p$ be an odd prime with $(3, p+1)=1$. Then there is a localization $U_{3}(p) \hookrightarrow G_{2}(p)$.

Proof The proof is similar to that of the preceding proposition. Apply also [Kle, Proposition 2.2] for the subgroup $K_{-}$. 
Proposition 4.4 There is a localization ${ }^{3} D_{4}(p) \hookrightarrow F_{4}(p)$ for any prime $p$.

Proof We have $\operatorname{Out}\left(F_{4}(2)\right) \cong C_{2}$ while for an odd prime $p, F_{4}(p)$ is complete. On the other hand Out $\left({ }^{3} D_{4}(p)\right)$ is cyclic of order 3. By [LS, Proposition 7.2] there are exactly $(2, p)$ conjugacy classes of subgroups isomorphic to ${ }^{3} D_{4}(p)$ in $F_{4}(p)$, fused by an automorphism if $p=2$. Applying Theorem 1.4, we see that the inclusion ${ }^{3} D_{4}(p) \hookrightarrow \operatorname{Aut}\left({ }^{3} D_{4}(p)\right) \hookrightarrow F_{4}(p)$ given by [LS, Table 1, p.300] is a localization.

We have seen various localizations involving sporadic groups in the proof of the main theorem. We give here further examples.

We start with the five Mathieu groups. Recall that the Mathieu groups $M_{12}$ and $M_{22}$ have $C_{2}$ as outer automorphism groups, while the three other Mathieu groups are complete. The inclusions $M_{11} \hookrightarrow M_{23}$ and $M_{23} \hookrightarrow M_{24}$ are localizations by Corollary 1.6. We have already seen in Section 3 that the inclusion $M_{11} \hookrightarrow M_{12}$ is a localization. The inclusion $M_{12} \hookrightarrow M_{24}$ is also a localization. Indeed $\operatorname{Aut}\left(M_{12}\right)$ is the stabilizer in $M_{24}$ of a pair of dodecads, the stabilizer of a single dodecad is a copy of $M_{12}$. Up to conjugacy, these are the only subgroups of $M_{24}$ isomorphic to $M_{12}$ and thus the condition (3') in Remark 1.5 is satisfied. Similarly $M_{22} \hookrightarrow M_{24}$ is also a localization, because $\operatorname{Aut}\left(M_{22}\right)$ can be identified as the stabilizer of a duad (a pair of octads) in $M_{24}$ whereas $M_{22}$ is the pointwise stabilizer (see [CCN, p.39 and p.94]). In short we have the following diagram, where all inclusions are localizations:

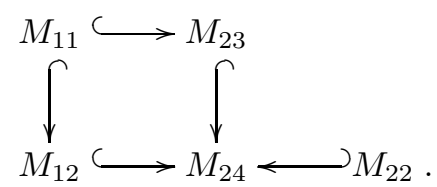

We consider next the sporadic groups linked to the Conway group $C o_{1}$. Inside $\mathrm{Co}_{1}$ sits $\mathrm{Co}_{2}$ as stabilizer of a certain vector $O A$ of type 2 and $\mathrm{Co}_{3}$ as stabilizer of another vector $O B$ of type 3 . These vectors are part of a triangle $O A B$ and its stabilizer is the group $H S$, whereas its setwise stabilizer is $\operatorname{Aut}(H S)$. The Conway groups are complete, the smaller ones are maximal simple subgroups of $C o_{1}$ and there is a unique conjugacy class of each of them in $C o_{1}$ as indicated in the ATLAS [CCN, p.180]. Hence $\mathrm{Co}_{2} \hookrightarrow \mathrm{Co}_{1}$ and $\mathrm{Co}_{3} \hookrightarrow \mathrm{Co}_{1}$ are localizations by Corollary 1.6. Likewise the inclusions $\mathrm{HS} \hookrightarrow \mathrm{Co}_{2}$ and $\mathrm{McL} \hookrightarrow \mathrm{Co}_{3}$ are also localizations: They factor through their group of automorphisms, since for example Aut $(M c L)$ is the setwise stabilizer of a triangle of type 223 in the Leech lattice, a vertex of which is stabilized by $\mathrm{Co}_{3}$. Finally, $M_{22} \hookrightarrow H S$ is a localization for similar reasons, since any automorphism of $M_{22}$ can be seen as an automorphism of the Higman-Sims graph (cf. [BJL, Theorem 8.7 p.273]). We 
get here the following diagram of localizations:

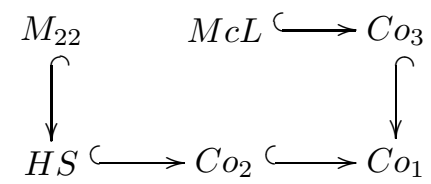

Some other related localizations are $M_{23} \hookrightarrow \mathrm{Co}_{3}, M_{23} \hookrightarrow \mathrm{Co}_{2}$ and $M_{11} \hookrightarrow H S$.

We move now to the Fischer groups and Janko's group $J_{4}$. The inclusion $T \hookrightarrow F i_{22}$ is a localization (both have $C_{2}$ as outer automorphism groups) as well as $M_{12} \hookrightarrow F i_{22}$, and $A_{10} \hookrightarrow F i_{22}$. Associated to the second Fischer group, we have a chain of localizations

$$
A_{10} \hookrightarrow S_{8}(2) \hookrightarrow F i_{23} .
$$

By [KPW, Theorem 1] the inclusion $A_{12} \hookrightarrow F i_{23}$ is also a localization. Moreover $M_{11} \hookrightarrow J_{4}$ and $M_{23} \hookrightarrow J_{4}$ are localizations by Corollaries 6.3 .2 and 6.3.4 in KW1.

Let us list now without proofs a few inclusions we know to be localizations. We start with two examples of localizations of alternating groups: $A_{12} \hookrightarrow H N$, and $A_{7} \hookrightarrow S u z$ by WS, Section 4.4]. Finally we list a few localizations of Chevalley groups: $L_{2}(8) \hookrightarrow S_{6}(2), L_{2}(13) \hookrightarrow G_{2}(3), L_{2}(32) \hookrightarrow J_{4}$ (by [KW1, Proposition 5.3.1]), $U_{3}(3) \hookrightarrow S_{6}(2),{ }^{3} D_{4}(2) \hookrightarrow T h, G_{2}(5) \hookrightarrow L y, E_{6}(2) \hookrightarrow E_{7}(2)$, and $E_{6}(3) \hookrightarrow E_{7}(3)$. The inclusion $E_{6}(q) \hookrightarrow E_{7}(q)$ is actually a localization if and only if $q=2$ or $q=3$ by [LS, Table 1]. The main theorem in [SWW] states that $S z(32) \hookrightarrow E_{8}(5)$ is a localization. There is a single conjugacy class of subgroups isomorphic to $S z(32)$ in $E_{8}(5)$, and $\operatorname{Out}(S z(32))$ is cyclic of order 5 .

We have seen a great deal of localizations of finite simple groups, and one could think at this point that they abound in nature. This is of course not so: During our work on this paper, we came across many more inclusions of simple groups that are not localizations. They do not appear here for obvious reasons. On the other hand, our list is certainly far from being complete. It would be nice for example to find other infinite families of localizations among groups of Lie type and to determine if they are connected to the alternating groups. Another interesting task would be to find out which simple groups are local with respect to a given localization $i: H \hookrightarrow G$, or even better to compute the localization of other simple groups with respect to $i$. Will they still be finite simple groups? 


\section{References}

[BJL] Beth, T., Jungnickel, D., and Lenz, H., Design Theory, Cambridge University Press, 1986.

[Bou] Bousfield, A. K., Constructions of factorization systems in categories, J. Pure Appl. Algebra 9 (1976/77), no. 2, 207-220.

[Car] Carter, R. W., Simple groups of Lie type, Pure and Applied Mathematics 28, John Wiley \& Sons, London-New York-Sydney, 1972.

[Cas] Casacuberta, C., On structures preserved by idempotent transformations of groups and homotopy types, in: Crystallographic Groups and Their Generalizations (Kortrijk, 1999), Contemp. Math. 262, Amer. Math. Soc., Providence, 2000, 39-68.

[CCN] Conway, J.H., Curtis, R.T., Norton, S.P., Parker, R.A., and Wilson, R.A., Atlas of finite groups, Clarendon Press, Oxford, 1985.

[Coo] Cooperstein, B. N., Minimal degree for a permutation representation of a classical group, Israel J. Math. 30 (1978), no. 3, 213-235.

[DM] Dixon, J. D, and Mortimer, B., Permutation groups, Graduate Texts in Mathematics 163, Springer-Verlag, Berlin-Heidelberg-New York, 1996.

[Far] Farjoun, E. D., Cellular spaces, null spaces and homotopy localization, Lecture Notes in Math. 1622, Springer-Verlag, Berlin-Heidelberg-New York, 1996.

[GRS] Göbel, R., Rodríguez, J. L., and Shelah, S., Large localizations of finite simple groups, to appear in J. Reine Angew. Math.

[GS] Göbel, R. and Shelah, S, Localizations of groups, to appear in Comm. Algebra (2001).

[Gor] Gorenstein, D., Finite simple groups. An introduction to their classification, The University Series in Mathematics, Plenum Press, New York - London, 1982.

[GLS] Gorenstein, D., Lyons, R., and Solomon, R., The classification of the finite simple groups. Number 3. Part I. Chapter A. Almost simple K-groups, Mathematical Surveys and Monographs, American Mathematical Society, Providence, RI, 1998

[Hup] Huppert, B., Endliche Gruppen I, Springer-Verlag, Berlin-Heidelberg-New York, 1967. 
[Kle] Kleidman, P. B., The maximal subgroups of the Chevalley groups $G_{2}(q)$ with $q$ odd, the Ree groups ${ }^{2} G_{2}(q)$, and their automorphism groups, J. Algebra 117 (1988), no. 1, 30-71.

[KPW] Kleidman, P. B., Parker, R. A., and Wilson, R. A., The maximal subgroups of the Fischer group $\mathrm{Fi}_{23}$, J. London Math. Soc. (2) 39 (1989), no. $1,89-101$.

[KW1] Kleidman, P. B., and Wilson, R. A., The maximal subgroups of $J_{4}$, Proc. London Math. Soc. (3) 56 (1988), no. 3, 484-510.

[KW2] Kleidman, P. B., and Wilson, R. A., $J_{3}<E_{6}(4)$ and $M_{12}<E_{6}(5)$, J. London Math. Soc. (2) 42 (1990), no. 3, 555-561.

[Lib1] Libman, A., A note on the localization of finite groups, J. Pure Appl. Algebra 148 (2000), no. 3, 271-274.

[Lib2] Libman, A., Cardinality and nilpotency of localizations of groups and $G$ modules, Israel J. Math. 117 (2000), 221-237.

[LS] Liebeck, M. W., and Saxl, J., On the orders of maximal subgroups of the finite exceptional groups of Lie type, Proc. London Math. Soc. (3) 55 (1987), no. 2, 299-330.

[LW] Linton, S. A., and Wilson, R. A., The maximal subgroups of the Fischer groups $\mathrm{Fi}_{24}$ and $\mathrm{Fi}_{24}^{\prime}$, Proc. London Math. Soc. (3) 63 (1991), no. 1, 113-164.

[RSV] Rodríguez, J. L., Scherer, J., and Viruel, A., Localization, Schur multipliers, and outer automorphisms, preprint 2001.

[Rot] Rotman, J. J., An introduction to the theory of groups, Fourth edition. Graduate Texts in Mathematics 148, Springer-Verlag, Berlin-HeidelbergNew York, 1995.

[SWW] Saxl, J., Wales, D. B., and Wilson, R. A., Embedings of $S z(32)$ in $E_{8}(5)$, Bull. London Math. Soc. 32 (2000), no. 2, 196-202.

[WS] Wilson, R. A., The complex Leech lattice and maximal subgroups of the Suzuki group, J. Algebra 84 (1983), no. 1, 151-188.

[Wil1] Wilson, R. A., The maximal subgroups of the O'Nan group, J. Algebra 97 (1985), no. 2, 467-473.

[Wil2] Wilson, R. A., The maximal subgroups of the Baby Monster, I, J. Algebra 211 (1999), no. 1, 1-14. 